\title{
ABSORÇÃO FOLIAR, CAULINAR E RADICULAR DOS INSETICIDAS PYMETROZINE E FLONICAMID NO CONTROLE DO PULGÃO APHIS GOSSYPII GLOVER, 1877 (HEMIPTERA: APHIDIDAE) EM ALGODOEIRO
}

\section{P.R.B. da Fonseca, R.P. Fortunato, I.S. de Lima Junior, T.F. Bertoncello, P.E. Degrande}

Universidade Federal da Grande Dourados, Faculdade de Ciências Agrárias, Rod. Dourados-Itahum, km 12, CEP 79804-970, Dourados, MS, Brasil. E-mail: paulodegrande@ufgd.edu.br

\section{RESUMO}

\begin{abstract}
O pulgão Aphis gossypii Glover, 1877 (Hemiptera: Aphididae) é uma praga da cultura do algodão e quando não controlado pode reduzir a produtividade e a qualidade da fibra. A descoberta de novos grupos químicos de inseticidas para o controle do inseto traz perspectivas novas de manejo, e dentre os aficidas recentemente descobertos destacam-se pymetrozine e flonicamid. Este trabalho teve como objetivo avaliar a eficiência destes dois inseticidas no controle de A. gossypii quando absorvidos pela folha, caule e raiz de algodoeiro. Utilizou o delineamento experimental fatorial $(2 \times 3)$ com 5 repetições, em blocos casualizados. Cada parcela foi constituída de um vaso com duas plantas, mantidos sob condições de casa-de-vegetação, no ano de 2005. Avaliou-se a densidade populacional do pulgãonas folhas durante dezesseis dias após a aplicação dos tratamentos. Os resultados permitem concluir: (1) flonicamid apresentou eficiência de controle quando absorvido pelo caule e pelas folhas; (2) pymetrozine apresentou eficiência de controle através da absorção foliar; (3) a rota de absorção radicular não propiciou controle satisfatório para ambos aficidas; e, (4) em aplicação foliar os dois inseticidas não diferiram entre si quanto ao controle do pulgão-do-algodoeiro.
\end{abstract}

PALAVRAS-CHAVE: Folha, caule, raiz, controle químico, translocação.

\section{ABSTRACT}

LEAF, STEM AND ROOT ABSORPTION OF PYMETROZINE AND FLONICAMID TO CONTROL THECOTTON APHID APHIS GOSSYPIIGLOVER, 1877(HEMIPTERA: APHIDIDAE). Aphis gossypii Glover, 1877 (Hemiptera: Aphididae) is a cotton pest, and if not controlled can reduce yield and fiber quality. The discovery of new chemical groups of insecticides brings new perspectives to IPM; pymetrozine and flonicamid are examples of new launched pesticides. This study was carried out to evaluate the efficacy of these two insecticides to control A. gossypii when they were absorbed by cotton root, stem and leaves. The statistical design was a randomized factorial $(2 \times 3)$ with five replications in blocks. Each plot consisted of a pot with two plants, kept under greenhouse conditions, in 2005. We evaluated the population density of aphids on the leaves during sixteen days after treatment. The results show: (1) flonicamid has efficiency when absorbed by stem and leaves, (2) pymetrozine efficacy is through foliar uptake, (3) the root absorption do not provide satisfactory control for both insecticides, and, (4) both insecticides control the aphid-cotton when is applied on leaves.

KEY WORDS: Leaf, stem, root, chemical control, translocation.

Opulgão Aphis gossypii Glover, 1877 (Hemiptera: Aphididae) éuma das primeiras pragas que ocorrena cultura do algodão logo após a germinação, sugando a seiva das plantas (GALlo et.al., 2002). Adultos e ninfas das colônias que normalmenteficam na página inferior das folhas, brotações, partes macias do caule, brácteas e demais tecidos tenros do algodoeiro sugam continuamente a seiva do floema (DEGRANDE, 1998; 2003), provocando encarquilhamento das folhas, deformação de brotos (NAKANO; SOUZA JunIOR, 2001), e paralisando o crescimento da planta. Segundo
Calcagnolo; Sauer (1954), A. gossypii quando não controlado pode reduzir em até $44 \%$ a produção de algodão. Além disso, danos indiretos podem ocorrer pela deposição de honeydew nas folhas e nos capulhos, onde se desenvolve a fumagina (Gondim et al., 1999) ou pode melar as fibras. Em variedades suscetíveis ao mosaico-das-nervuras, o pulgão é considerado a principal praga da cultura por ser vetor deste vírus (FreIre, 1999; Costa et al., 1997).

A descoberta do flonicamid, um novo grupo de inseticida sistêmico, veio trazer novas perspectivas 
no controle do pulgão, por afetar a glândula salivar do inseto, paralisandoa sua alimentaçãoe, em consequência a inoculação do vírus do mosaico-das-nervuras (NAKANO et al., 2003). O sítio de ação do flonicamid ocorre nos canais de potássio tipo A dos terminais pré-sinápticos, um comportamento completamente diferente daquele dos ciclodienos, neonicotinoides, tioureias ecarbamatos, mostrando ser uma alternativa de rotação de modo de ação com os novos e antigos aficidas do mercado. O pymetrozine também é um bloqueador seletivo de alimentação (IRAC, 2010).

Este trabalho teve por objetivo avaliar a eficácia do flonicamid (Turbine ${ }^{\circledR} 500$ WG) e do pymetrozine (Chess ${ }^{\circledR} 500$ WG) absorvidos pela folha, caule e raiz do algodoeiro no controle do A. gossypii.

$O$ experimento foi realizado na Universidade Federal da Grande Dourados (UFGD), Faculdade de Ciências Agrárias (FCA), no Município de Dourados, MS, sob condições de casa-de-vegetação, no ano de 2005. Para tal, foi utilizado o esquema experimental bifatorial com doisinseticidas, três locais de aplicação e uma testemunha não tratada, em blocos casualisados com cinco repetições. Cada parcela consistiu de um vaso com duas plantas de algodoeiro.
Os algodoeiros foram cultivados em vasos, obtidos a partir da semeadura de quatro sementes da variedade DeltaOpal ${ }^{\circledR}$ por parcela, no dia 5/11, e foi efetuado o desbaste logo ao emergirem, deixando as duas plantas vigorosas e uniformes em tamanho e morfologia. Quando as plantas encontravam-se no estádio V3, de acordo com a escala proposta por MARUR; RUANO (2004), elas foram infestadas com a praga. Para a infestação, foram coletadas folhas de algodoeiro com afídeos numa lavoura e foi feita a liberação dos insetos nas plantas dos vasos, colonizando-as com os pulgões.

Após a constatação da formação das colônias e da avaliação prévia à aplicação dos tratamentos, os inseticidas foram aplicados através de uso de pincéis com cerdas macias, nas folhas e nos caules, e com uma seringa no solo e junto às raízes, nos respectivos tratamentos, sendo usados flonicamid (Turbine ${ }^{\circledR} 500$ WG) na dose de $0,15 \mathrm{~kg} /$ ha e pymetrozine (Chess ${ }^{\circledR} 500$ WG) com 0,2 kg/ha, na diluição de 400 litros de calda por hectare. Pincéis diferentes foram utilizados para cada local da aplicação e cada produto. Em todos os tratamentos, cada plantarecebeu dois mililitros decalda inseticida, independentemente da forma de aplicação.

Tabela 1 - Análise da variância para eficiência de controle de Aphis gossypii em diferentes datas após aplicação dos tratamentos. Dados transformados pela fórmula $\sqrt{\mathrm{X}+0,5}$, Dourados, MS, 2005.

\begin{tabular}{|c|c|c|c|c|c|c|c|}
\hline \multicolumn{4}{|c|}{$1 \mathrm{DAA}$} & \multicolumn{4}{|c|}{$2 \mathrm{DAA}$} \\
\hline Fatores & G.L. & Q.M. & Fc. & Fatores & G.L. & Q.M. & Fc. \\
\hline Inseticida & 1 & 6,47 & 0,867 & Inseticida & 1 & 4,53 & 0,755 n.s. \\
\hline Local & 2 & 23,84 & 3,195 & Local & 2 & 43,6 & 7,263 n.s. \\
\hline Interação & 2 & 13,09 & 0,759 & Interação & 2 & 0,923 & 0,153 n.s. \\
\hline Erro & 24 & -- & -- & Erro & 24 & -- & -- \\
\hline \multicolumn{4}{|c|}{$3 \mathrm{DAA}$} & \multicolumn{4}{|c|}{$4 \mathrm{DAA}$} \\
\hline Fatores & G.L. & Q.M. & Fc. & Fatores & G.L. & Q.M. & Fc. \\
\hline Inseticida & 1 & 5,55 & 0,955 s. & Inseticida & 1 & 29,45 & 5,519 s. * \\
\hline Local & 2 & 81,16 & 6,975 s. & Local & 2 & 49,72 & 9,319 s. \\
\hline Interação & 2 & 6,35 & 0,546 n.s. & Interação & 2 & 10,72 & 2,009 n.s. \\
\hline Erro & 24 & -- & -- & Erro & 24 & -- & -- \\
\hline \multicolumn{4}{|c|}{$5 \mathrm{DAA}$} & \multicolumn{4}{|c|}{$6 \mathrm{DAA}$} \\
\hline Fatores & G.L. & Q.M. & Fc. & Fatores & G.L. & Q.M. & Fc. \\
\hline Inseticida & 1 & 63,86 & 11,763 s. & Inseticida & 1 & 7,52 & 1,472 n.s. \\
\hline Local & 2 & 57,85 & 10,655 s. & Local & 2 & 48,57 & 9,506 s. \\
\hline Interação & 2 & 24,91 & 4,588 s. & Interação & 2 & 19,66 & $3,847 \mathrm{~s}$ \\
\hline Erro & 24 & -- & -- & Erro & 24 & -- & -- \\
\hline \multicolumn{4}{|c|}{$7 \mathrm{DAA}$} & \multicolumn{4}{|c|}{9 DAA } \\
\hline Fatores & G.L. & Q.M. & Fc. & Fatores & G.L. & Q.M. & Fc. \\
\hline Inseticida & 1 & 1,861 & 0,429 n.s. & Inseticida & 1 & 19,72 & 3,507 n.s. \\
\hline Local & 2 & 41,63 & 9,605 s. & Local & 2 & 84,38 & 15,007 s. \\
\hline Interação & 2 & 15,53 & 3,583 s. & Interação & 2 & 34,662 & 6,158 s. \\
\hline Erro & 24 & -- & -- & Erro & 24 & -- & -- \\
\hline \multicolumn{4}{|c|}{$12 \mathrm{DAA}$} & \multicolumn{4}{|c|}{16 DAA } \\
\hline Fatores & G.L. & Q.M. & Fc. & Fatores & G.L. & Q.M. & Fc. \\
\hline Inseticida & 1 & 51,2 & $10,181 \mathrm{~s}$. & Inseticida & 1 & 13,14 & 1,338 n.s. \\
\hline Local & 2 & 55,26 & 10,989 s. & Local & 2 & 19,14 & 1,949 n.s. \\
\hline Interação & 2 & 11,13 & 2,213 n.s. & Interação & 2 & 12,48 & 1,271 n.s. \\
\hline Erro & 24 & -- & -- & Erro & 24 & -- & -- \\
\hline
\end{tabular}

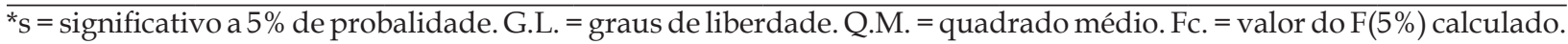


Ao todo foram feitas onze amostragens, sendo: uma prévia, na véspera da aplicação (15/12), e mais dez avaliações realizadas 1, 2, 3, 4, 5, 6, 7, 9, 12 e 16 dias após os tratamentos. Nestas avaliações foram contados os pulgões (adultos e ninfas) vivos encontrados nas duas plantas da parcela. Para o cálculo da porcentagem de eficiência de cada tratamento foi utilizada a Fórmula de Henderson; Tilton (1955) que considera a população inicial obtida na avaliação prévia. A eficiência de controle foi submetida à análise de variância e ao teste $\mathrm{F}$ de significância $(\alpha=0,05)$. Para a representação gráfica utilizou-se o erro padrão (5\%).

No primeiro dia após a aplicação, observou-se uma eficiência de controle inferior a $80 \%$ em todos os tratamentos; este nível mínimo é referido por GARCIA (1999) para minimizar o risco de evolução da resistência das pragas aos inseticidas e falhas no controle. Assim, o efeito de choque de ambos inseticidas inexistiu independente do local de aplicação (Fig. 1). As diferenças entre os tratamentos começaram a surgir após o segundo dia da aplicação, inclusive com efeito significativo para interação entre inseticidas e locais de aplicação até os 12 dias após aplicação (Tabela 1).

Em continuidade, éimportante apontar que ocorreu um maior controle de pulgões quando a aplicação foi realizada na folha, ficando acima de $90 \%$ para ambos os produtos após três dias após aplicação (Fig. 1C), o que está relacionado ao modo de ação destes produtos, pois segundo Nicholsonetal. (1995), com a aplicação de pymetrozine os pulgões param de sugar a planta e morrem, por não se alimentarem, mas necessita de tempo para que isso ocorra.

Os tratamentos comflonicamid aplicados no caule e folhas apresentaram controles semelhantes (Fig. 2B). O pymetrozine apresentou efetividade apenas quando aplicado na folha (Fig. 2A). Esses resultados evidenciam a maior mobilidade de flonicamid pelo xilema quando comparado ao pymetrozine (Fig.1B), e que ambos os inseticidas são translocados pelo floema, o que confirmam os estudos realizados por WYSS; BoLSINGER (1999), os quais demonstraram que após aplicação de pymetrozine nas folhas, regiões de crescimento da planta estavam protegidas pelo composto importado das folhas mais velhas.
(A)

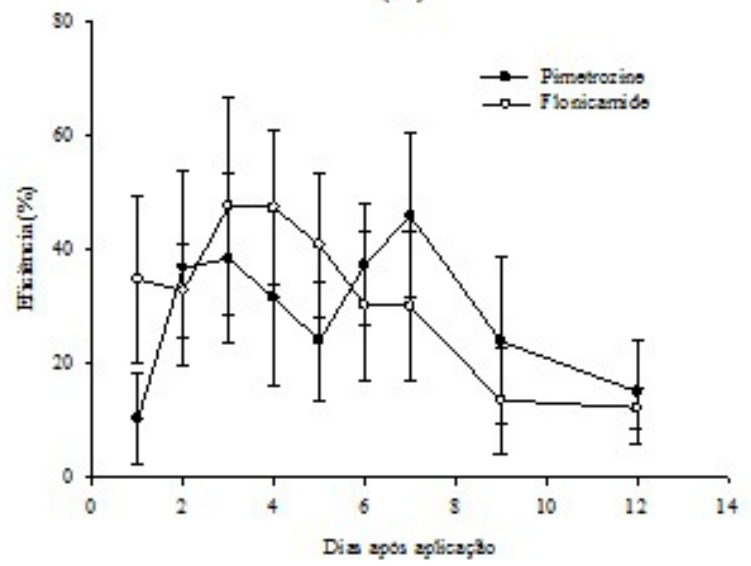

(C)

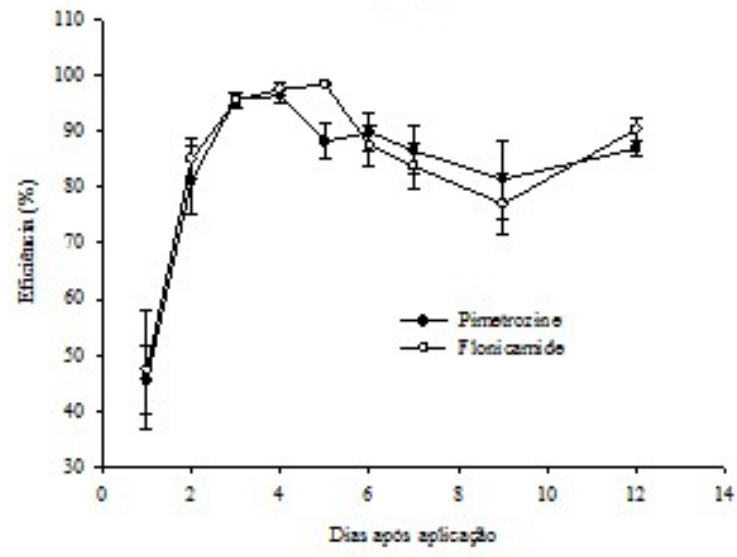

(B)

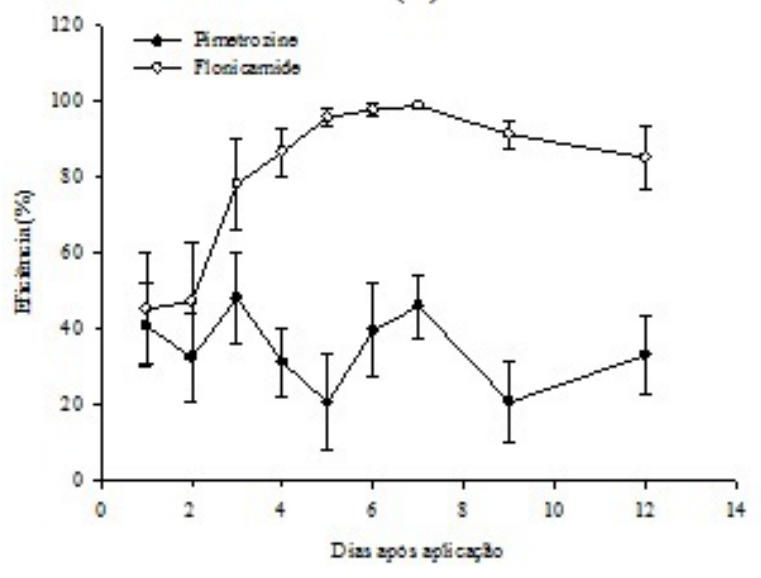

Fig. 1 - Eficiência de controle de A. gossypii em aplicação no solo (A), caule (B) e folha (C). Erro padrão (5\%). Dourados, MS, 2005. 
(A)

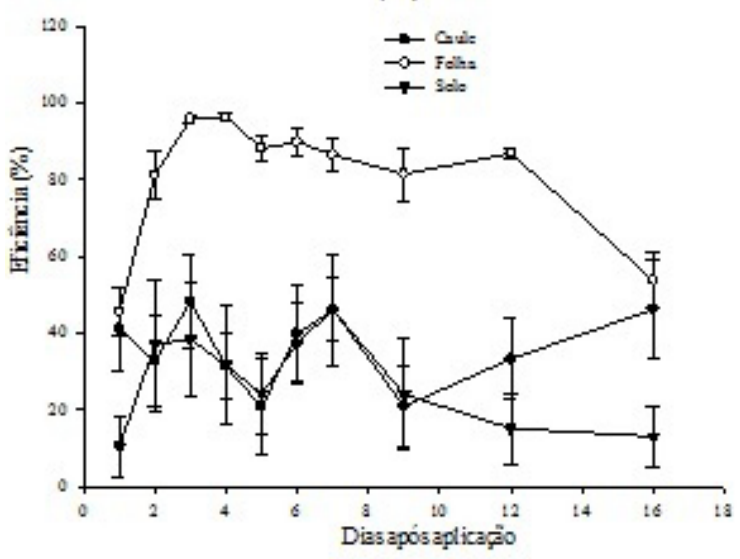

(B)

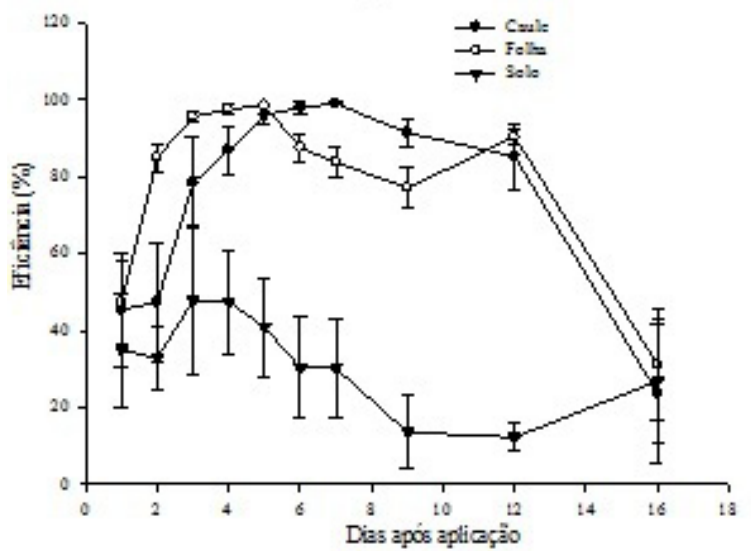

Fig. 2 - Eficiência de controle de Aphis gossypii com aplicação de pimetrozine (A) e flonicamide (B). Erro padrão (5\%). Dourados, MS. 2005.

O controle mais efetivo perdurou até 7 dias após aplicação, reduzindo a eficiência aos 9 dias que se manteve em declínio até a última avaliação (Figs. 1 e 2). Nogueira et al. (2003), avaliando a eficiência de flonicamid (150 g. p.c/ha) aplicados em algodoeio no campo, constataram controle do pulgão acima de $90 \%$ até os 12 dias após aplicação. Já a rota de absorção radicular não propiciou controle satisfatório para ambos aficidas testados (Fig. 1A).

\section{CONCLUSÕES}

O inseticida flonicamid controlou o A. gossypii quando aplicado na folha e no caule, enquanto o pymetrozine apresentou eficiência em aplicação na folha. Quando aplicados na folha, pymetrozine e flonicamid não diferiram entre si quanto ao controle de A. gossypii. A rota de absorção radicular não propiciou controle satisfatório para ambos aficidas.

\section{REFERÊNCIAS}

CALCAGNOLO, G.; SAUER, H.F.G. A influência do ataque dos pulgões (Aphis gossypii Glover, 1876, Hom. Aphididae) na produção da cultura do algodoeiro. Archivos do Instituto Biológico, São Paulo, v.21, p.85-99, 1954.

COSTA, A.S.; JULIATTI, F.C.; RUANO, O. Algodão (Gossypium hirsutum L.): doenças causadas por vírus. In: VALE, F.X.R. do; ZAMBOLIM, L. (Ed.). Controle de doenças de plantas: grandes culturas. Viçosa: UFV, 1997. v.2, cap.12, p.571-582.

DEGRANDE, P.E. Guia prático de controle das pragas do algodoeiro. Dourados: UFMS. 1998. 60p.
DEGRANDE, P.E. Os pulgões do algodoeiro. Correio Agrícola, n.2, p.18-20, 2003.

FREIRE, E.C. Doença azul tem solução. Cultivar Grandes Culturas, v.1, n.1, p.64-65, 1999.

GALLO, D.; NAKANO, O.; NETO, S.S.; CARVALHO, R.P.L; BAPTISTA, G.C.; BERTI FILHO, E.; PARRA, J.R.P.; ZUCCHI, R.A.; ALVES, S.B.; VENDRAMIN, J.D.; LOPES, J.R.S.; OMOTO, C. Entomologia agrícola. 3.ed. Piracicaba: FEALQ, 2002. p.401-403.

GARCIA, F.R.M. Zoologia agrícola: manejo ecológico de pragas. Porto Alegre: Rígel, 1999. 248p.

GOMES, F.P. Curso de estatística experimental. 12.ed. São Paulo: Nobel, 1987. 467p.

GONDIM, D.M.C.; BELOT, J.L.; SILVIE, P.; PETIT, N. Manual de identificação das pragas, doenças, deficiências minerais e injúrias do algodoeiro no Brasil. 3.ed. Cascavel, PR: COODETEC/CIRAD-CA, 1999. 120p.

HENDERSON, C.F.; TILTON. E.W. Tests with acaricides against the brown wheat mite. Journal of Economic Entomology, v.48, p.157-161, 1955.

IRAC - Insecticide Resistance Action Committee. Mode of Action Classification: Insecticide Resistance Management. Brochure, version 1, 2010. 20p.

MARUR, C.J.; RUANO, O. Escala do algodão: um método para determinação de estádios de desenvolvimento do algodoeiro herbáceo. Piracicaba: Potafós, 2004. p.3-4.

(Informações Agronômicas, n.105).

NAKANO, O.; SANTOS, F.G. dos; SAZAKI, C.S.S. F-1785 novo inseticida sistêmico para controle do pulgão do algodoeiro - Aphis gossypii Glover, 1877 (Hemiptera - Aphididae). In: CONGRESSO BRASILEIRO DE ALGODÃO, 4 ., 
Goiânia. Anais. 2003, Goiânia: Embrapa, 2003. 1-CDRom.

NAKANO, O.; SOUZA JUNIOR, J.A. de. Efeito do tratamento de sementes com os inseticidas Pirâmide (acetamipride) e thiamethoxan para controlar o pulgão (Aphis gossypii), na cultura do algodão. In: CONGRESSO BRASILEIRO DE ALGODÃO, 3., 2001, Campo Grande. Anais. Campina Grande: EMBRAPA-CNPA, 2001. p.215-218.

NICHOLSON, W.F.; SENN, R.; FLUECKIGER, C.R.; FUOG, D. Pymetrozine: a novel compound for control of whiteflies. In: GERLING, D.; MAYER, D. (Ed.). Bemisia: taxonomy, biology, damage, control and management. Andover: Intercept, 1995. p.635-639.
NOGUEIRA R.F.; RODRIGUES, A.L.L.; RIBEIRO, J.F.; BARROS, R.; DEGRANDE, P.E. Desempenho do inseticida flonicamid em pulverização foliar no controle do pulgão Aphis gossypii em algodoeiro. In: CONGRESSO BRASILEIRO DE ALGODÃO, 4., 2003, Goiânia. Anais. Goiânia: Embrapa, 2003. 1-CD-Rom.

WYSS, P.; BOLSINGER, M. Translocation of pymetrozine in plants. Pesticide Science, v.50, p.195-202, 1999.

Recebido em 6/8/09

Aceito em 13/12/10 